\title{
Diffusion tensor imaging quantifying the severity of chronic hepatitis in rats
}

\author{
Mengping Huang ${ }^{\dagger}$, Xin Lư ${ }^{\dagger}$ Xiaofeng Wang and Jian Shu ${ }^{*}$ (D)
}

\begin{abstract}
Background: Diffusion tensor imaging (DTI) is mainly used for detecting white matter fiber in the brain. DTI was applied to assess fiber in liver disorders in previous studies. However, the data obtained have been insufficient in determining if DTI can be used to exactly stage chronic hepatitis. This study assessed the value of DTI for staging of liver fibrosis (F), necroinflammatory activity (A) and steatosis (S) with chronic hepatitis in rats.

Methods: Seventy male Sprague-Dawley rats were divided into a control group $(n=10)$ and an experimental group $(n=60)$. The rat models of chronic hepatitis were established by abdominal subcutaneous injections of $40 \%$ $\mathrm{CCl}_{4}$. All of the rats underwent 3.0 T MRI. Regions of interest (ROIs) were subjected to DTI to estimate the MR parameters (rADC value and FA value). Histopathology was used as the reference standard. Multiple linear regression was used to analyze the associations between the MR parameters and pathology. The differences in the MR parameters among the pathological stages were evaluated by MANOVA or ANOVA. The LSD test was used to test for differences between each pair of groups. ROC analysis was also performed.

Results: The count of each pathology was as follows: F0( $(n=15), \mathrm{F} 1(n=11), \mathrm{F} 2(n=6), \mathrm{F} 3(n=9), \mathrm{F} 4(n=6) ; \mathrm{A} 0(n=8)$, $A 1(n=16), A 2(n=16), A 3(n=7) ; S 0(n=10), S 1(n=7), S 2(n=3), S 3(n=11), S 4(n=16)$. The rADC value had a negative correlation with liver fibrosis $(r=-0.392, P=0.008)$ and inflammation $(r=-0.359, P=0.015)$. The FA value had a positive correlation with fibrosis $(r=0.409, P=0.005)$. Significant differences were found in the FA values between F4 and F0 F3 $(P=0.03)$, while no significant differences among F0 $\sim F 3$ were found $(P>0.05)$. The AUC of the FA value differentiating F4 from F0 F3 was $0.909(p<0.001)$ with an $83.3 \%$ sensitivity and an $85.4 \%$ specificity when the FA value was at the cut-off of $588.089\left(\times 10^{-6} \mathrm{~mm}^{2} / \mathrm{s}\right)$.
\end{abstract}

Conclusion: The FA value for DTI can distinguish early cirrhosis from normal, mild and moderate liver fibrosis, but the rADC value lacked the ability to differentiate among the fibrotic grades. Both the FA and rADC values were unable to discriminate the stages of necroinflammatory activity and steatosis.

Keywords: Chronic hepatitis, Liver fibrosis, Diffusion tensor imaging, Average diffusion coefficient, Fractional anisotropy

\footnotetext{
* Correspondence: shujiannc@163.com

'Note: Mengping Huang and Xin Lu had materially participated in the

research and made the equal contribution, so they all ranked as the co-first authors.

Department of Radiology, The Affiliated Hospital of Southwest Medical University, 25 Taiping Street, Luzhou, Sichuan 646000, People's Republic of China
}

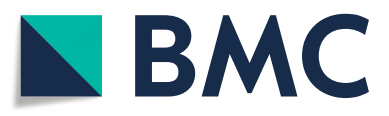

(C) The Author(s). 2020 Open Access This article is licensed under a Creative Commons Attribution 4.0 International License, which permits use, sharing, adaptation, distribution and reproduction in any medium or format, as long as you give appropriate credit to the original author(s) and the source, provide a link to the Creative Commons licence, and indicate if changes were made. The images or other third party material in this article are included in the article's Creative Commons licence, unless indicated otherwise in a credit line to the material. If material is not included in the article's Creative Commons licence and your intended use is not permitted by statutory regulation or exceeds the permitted use, you will need to obtain permission directly from the copyright holder. To view a copy of this licence, visit http://creativecommons.org/licenses/by/4.0/. The Creative Commons Public Domain Dedication waiver (http://creativecommons.org/publicdomain/zero/1.0/) applies to the data made available in this article, unless otherwise stated in a credit line to the data. 


\section{Background}

Chronic hepatitis is a typical chronic diffuse liver disease that can be caused by many factors [1]. The basic pathological changes associated with chronic hepatitis include hepatic inflammation, liver fibrosis and fatty infiltration and the disease can further develop into cirrhosis and even liver cancer and liver failure [2, 3]. Studies [4] have shown that early chronic hepatitis manifests as a dynamic and reversible lesion. Furthermore, early diagnosis and accurate staging of chronic hepatitis are clinically significant in evaluating the severity and progress of the disease. Percutaneous liver biopsy is considered the gold standard, but patients often reject this invasive technique, as they usually have no symptoms [5].

Several noninvasive methods have been presented chronic hepatitis, among which the most promising ones are ultrasound elastography [6] and magnetic resonance elastography [7]; however, the former is inadequate or unavailable in obese and abdominal dropsy patients and the latter is expensive. DTI is a mature magnetic resonance imaging (MRI) sequence developed on the basis of diffusion weighted imaging (DWI). Compared with unidirectional or three orthogonal directional DWI, DTI quantifies the diffusivity of water molecules by using six or more different directions of diffusion sensitive gradients, traces the fiber bundle shape, and visually reveals the microstructural characteristics of biological tissues. The DTI sequence produces an average diffusion coefficient (rADC) image, a fractional anisotropy (FA) image, a relative anisotropy image and the corresponding values (among which the rADC and FA values are widely used) [8].

DTI is mainly used to detect white matter fiber in the nervous system [9]. Some studies [10-13] have applied DTI for chronic hepatitis because fibrosis always emerges in liver damage. Previous studies [12, 13] have shown that the $\mathrm{CCl}_{4}$-induced liver fibrosis animal model is a mature technique and can mirror the pathophysiologic processes of fibrogenesis in humans [14]. However, insufficient data have been obtained. Our study assessed whether the rADC and FA values of DTI can help to distinguish the different stages of liver fibrosis, necroinflammatory activity and steatosis in rats with chronic hepatitis induced by abdominal subcutaneous injection of $\mathrm{CCl}_{4}$

\section{Methods}

\section{Establishment of a chronic hepatitis model in rats}

This study was approved by the Animal Ethics Committee of the Southwest Medical University. Seventy 7week-old male Sprague-Dawley rats weighing 150-200 g were randomly divided into a control group $(n=10)$ and an experimental group $(n=60)$. The rats were purchased from Animal Experimental Center of Southwest Medical
University. A chronic hepatitis model was induced in rats by abdominal subcutaneous injection of a $40 \% \mathrm{CCl}_{4}$ suspension (99.9\% carbon tetrachloride: vegetable oil $=4$ : 6) at a dose of $0.3 \mathrm{ml} / 100 \mathrm{~g}$ twice a week. The rats in the control group were injected with $0.9 \%$ sodium chloride at the same dose and in the same way. The animals were raised under standard conditions and had free access to food and water.

\section{MR imaging}

Five weeks after injecting $\mathrm{CCl}_{4}, 6$ to 10 rats for the test group and 1or 2 rats for the control group were randomly selected for an MRI scan every week. The rats were supinely fixed on boards under anesthesia during scanning by intraperitoneal injection of $1 \%$ pentobarbital sodium at a dose of $0.5 \mathrm{ml} / 100 \mathrm{~g}$.

The MR exams were performed on a $3.0 \mathrm{~T}$ MRI scanner(Achieva 3.0 T, Philips, Netherlands) using 8-channel knee coils. Spin-echo echo-planar imaging diffusion tensor imaging sequence; two b values $\left(0\right.$ and $\left.800 \mathrm{~s} / \mathrm{mm}^{2}\right)$; 15 diffusion gradient directions; TR, $3907 \mathrm{~ms}$; TE, $86 \mathrm{~ms}$; FOV, $100 \mathrm{~mm} \times 100 \mathrm{~mm}$, thickness, $2.0 \mathrm{~mm}$; NSA, 3; matrix, 240.

\section{Image analysis}

Two radiologists who were blinded to the pathological results used a postprocessing workstation (Philips Extended MR WorkSpace 2.6.3.4) to generate functional imaging maps (rADC image and FA image) and measure the quantitative indicators of the regions of interest (ROIs). Three circular ROIs per slice ranging from 5 $\mathrm{mm}^{2} \sim 10 \mathrm{~mm}^{2}$ were placed on two consecutive slices of the DTI images of $\mathrm{b}=0 \mathrm{~s} / \mathrm{mm}^{2}$ and were then copied to the same slices of rADC images and FA images (Fig.1). The mean values of the six ROIs measured by the two radiologists were estimated. The average value of the two radiologists' measurements for each specimen was utilized as the final measurement. Care was taken to avoid large vessels, and the edge of the ROI was at least $3 \mathrm{~mm}$ away from the border of the liver.

\section{Histopathological evaluation}

For the pathological evaluation, the rats were sacrificed by cervical dislocation immediately after the MRI scan. In addition, hematoxylin and eosin staining and Masson staining were performed. According to the METAVIR scoring system [15], hepatic fibrosis (F) was classified on a 5-point scale (F0: no fibrosis; F1: portal fibrosis without septa; F2: portal fibrosis with rare septa; F3: numerous septa without cirrhosis; F4: cirrhosis) and necroinflammatory activity (A) was classified on a 4point scale(A0: no activity; A1: mild activity; A2: moderate activity; A3: severe activity). Steatosis(S) is depended on the percentage of liver cells containing fat droplets as 

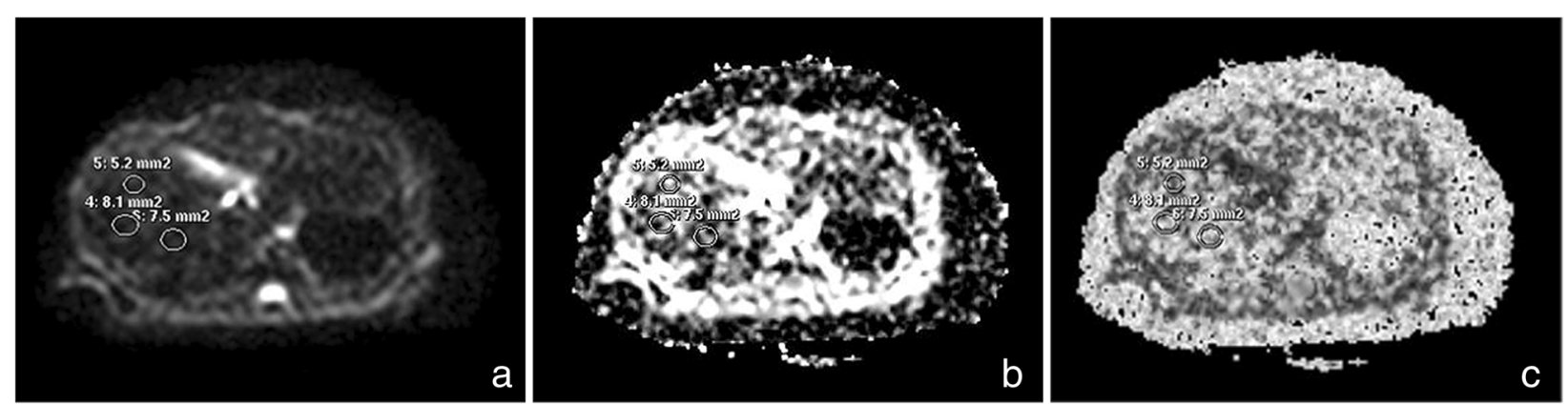

Fig. $1 \mathrm{rADC}$ and FA value calculation. a The three circular ROls ranging from $5 \mathrm{~mm}^{2} \sim 10 \mathrm{~mm}^{2}$ were placed on the DTI images at $\mathrm{b}=0 \mathrm{~s} / \mathrm{mm}^{2}$. $\mathbf{b}$ ROIs were copied to the rADC image, and the computer calculated the rADC values. c ROls were copied to the FA image, and the computer calculated the FA values

follows: S0(0-5\%), S1 (6-30\%), S2 (31-50\%), S3 (51$75 \%)$, and S4 (>75\%). The liver sections were assessed by two pathologists who did not know the radiologic outcome.

\section{Statistical analysis}

The intraclass correlation coefficient (ICC) was calculated to evaluate the reliability of the values measured by the two radiologists. Multiple linear regression (Enter model) analysis was used to examine the relationships between the MR parameters (rADC value and FA value) and pathological stage (necroinflammation, hepatic fibrosis and steatosis). The differences in the magnetic resonance parameters among the pathological stages were evaluated by a multi factor analysis of variance (MANOVA) or a one-way analysis of variance (ANOVA). The least significant difference (LSD) method was used to test the differences between each pair of groups. To evaluate the diagnostic performance of the FA value for the assessment of fibrosis stage, a receiver operating characteristic (ROC) curve was constructed. A $P$ value less than 0.05 was considered statistically significant.

\section{Result}

\section{General feature of the animal models and pathologic} results

Sixteen rats died in the experimental group during induction of the model. Five specimens in the experimental group and one specimen in the control group which had a low signal-to-noise ratio were eliminated despite taking measures to reduce artifacts. Finally, there were 47 specimens available including $8(8 / 10)$ control group rats and 39(39/60) chronic hepatitis rats. The statistical data were as follows: $\mathrm{F} 0(n=15), \mathrm{F} 1(n=11), \mathrm{F} 2(n=6)$, $\mathrm{F} 3(n=9), \mathrm{F} 4(\mathrm{n}=6) ; \mathrm{A} 0(n=8), \mathrm{A} 1(n=16), \mathrm{A} 2(\mathrm{n}=16)$, $\mathrm{A} 3(n=7) ; \quad \mathrm{S} 0(n=10), \quad \mathrm{S} 1(\mathrm{n}=7), \quad \mathrm{S} 2(n=3), \quad \mathrm{S} 3(\mathrm{n}=11)$, S4 $(\mathrm{n}=16)$. All the data are summarized in Table 1 .
Table 1 Distribution of rADC value and FA value in pathology of the liver

\begin{tabular}{|c|c|c|c|}
\hline Pathological stage & number & $\operatorname{rADC}\left(\times 10^{-6} \mathrm{~mm}^{2} / \mathrm{s}\right)$ & $\mathrm{FA}\left(\times 10^{-6} \mathrm{~mm}^{2} / \mathrm{s}\right)$ \\
\hline AOFOSO & 8 & $923.008 \pm 69.899$ & $505.421 \pm 34.550$ \\
\hline A1F0S1 & 2 & $885.900 \pm 19.351$ & $489.075 \pm 44.182$ \\
\hline A1F0S4 & 1 & 1167.383 & 372.433 \\
\hline A1F1S1 & 2 & $941.417 \pm 128.057$ & $455.658 \pm 118.688$ \\
\hline A1F1S4 & 4 & $852.388 \pm 150.181$ & $553.717 \pm 99.250$ \\
\hline A1F2SO & 1 & 875.067 & 582.250 \\
\hline $\mathrm{A} 1 \mathrm{~F} 2 \mathrm{~S} 3$ & 2 & $760.892 \pm 30.205$ & $526.508 \pm 55.425$ \\
\hline A1F2S4 & 2 & $839.900 \pm 47.588$ & $526.508 \pm 55.425$ \\
\hline A1F3S3 & 2 & $774.908 \pm 88.565$ & $535.308 \pm 140.184$ \\
\hline A2F0S4 & 1 & 852.233 & 405.000 \\
\hline A2F1S3 & 1 & 683.100 & 664.783 \\
\hline A2F1S4 & 3 & $795.483 \pm 128.615$ & $465.306 \pm 181.995$ \\
\hline $\mathrm{A} 2 \mathrm{~F} 3 \mathrm{~S} 1$ & 1 & 637.583 & 598.350 \\
\hline $\mathrm{A} 2 \mathrm{~F} 3 \mathrm{~S} 2$ & 2 & $818.750 \pm 26.729$ & $494.017 \pm 73.044$ \\
\hline $\mathrm{A} 2 \mathrm{~F} 3 \mathrm{~S} 3$ & 1 & 776.233 & 536.083 \\
\hline $\mathrm{A} 2 \mathrm{~F} 3 \mathrm{~S} 4$ & 1 & 866.550 & 582.167 \\
\hline $\mathrm{A} 2 \mathrm{~F} 4 \mathrm{SO}$ & 1 & 743.350 & 634.433 \\
\hline $\mathrm{A} 2 \mathrm{~F} 4 \mathrm{~S} 1$ & 2 & $651.425 \pm 28.555$ & $731.483 \pm 21.920$ \\
\hline $\mathrm{A} 2 \mathrm{~F} 4 \mathrm{~S} 2$ & 1 & 701.783 & 759.617 \\
\hline $\mathrm{A} 2 \mathrm{~F} 4 \mathrm{~S} 3$ & 2 & $774.775 \pm 94.599$ & $576.917 \pm 26.328$ \\
\hline A3FOS3 & 2 & $774.975 \pm 63.062$ & $530.283 \pm 58.266$ \\
\hline A3FOS4 & 1 & 763.033 & 606.250 \\
\hline A3F1S4 & 1 & 826.183 & 525.267 \\
\hline A3F2S4 & 1 & 736.933 & 544.250 \\
\hline A3F3S3 & 1 & 804.083 & 545.117 \\
\hline A3F3S4 & 1 & 803.050 & 566.138 \\
\hline
\end{tabular}

Abbreviations: A, necroinflammatory activity; $F$ fibrosis, $S$ steatosis, $r A D C$ average diffusion coefficient, $F A$ fractional anisotropy 


\section{MRI quantitative indicators}

The ICC of the rADC value was $0.852(P<0.001)$, and that of the FA value was $0.922(P<0.001)$. High measurement repeatability between the two observers indicated the clinical feasibility of this method.

The results of the multiple linear regression analysis are presented in Table 2 . The rADC value was correlated with fibrosis $(r=-0.392, P=0.008)$ and necroinflammatory activity $(r=-0.359, P=0.015)$, but not with steatosis $(P=0.452)$. The FA value was related to the degree of fibrosis $(r=0.409, P=0.005)$, but not to inflammatory $\operatorname{activity}(P=0.236)$ or steatosis $(P=0.115)$. Table 3 shows the rADC value and FA value of the different stages of liver fibrosis and indicates that the rADC value decreased with the severity of liver fibrosis while the FA value increased.

Based on the means from MANOVA, no significant differences were found among the stages of fibrosis $(F=$ 1.250, $P=0.309)$ or inflammatory activity $(F=1.487, P=$ $0.236)$ for the rADC value. When analyzed by ANOVA, the FA values among the different fibrosis groups were significantly different $(F=4.750, P=0.03)$. There was a significant difference in the FA value between F4 and F0 $\sim$ F3 $(P<0.05)$, while no significant differences among F0 $\sim$ F3 were found $(P>0.05)$. The area under the ROC curve (AUC) of the FA value that differentiated F4 from F0 F3 was $0.909(p<0.001)$ at a cut-off of $588.089(\times$ $10^{-6} \mathrm{~mm}^{2} / \mathrm{s}$ ), with an $83.3 \%$ sensitivity and an $85.4 \%$ specificity. Figure 2 shows FA images of the different liver fibrosis stages.

\section{Discussion}

Our study indicated that the rADC value was negatively related to hepatic fibrosis and necroinflammatory activity but not to steatosis. However, the rADC value was not significantly different among the stages of fibrosis or necroinflammatory activity. The FA value had a positive correlation with the degree of fibrosis, but no correlation with necroinflammatory activity or steatosis. The FA value of F4 was significantly different from those of F0 F3, while there were no significant differences among F0 $\sim \mathrm{F} 3$, which suggests that the liver $\mathrm{FA}$ value can

Table 2 Results of the regression analysis between quantitative indexes of DTI and pathology

\begin{tabular}{|c|c|c|c|c|c|c|}
\hline \multirow{2}{*}{$\begin{array}{l}\text { Pathologic } \\
\text { staging }\end{array}$} & \multicolumn{3}{|c|}{ rADC value } & \multicolumn{3}{|l|}{ FA value } \\
\hline & $B$ & $r$ & $P$ & $B$ & $r$ & $P$ \\
\hline $\bar{A}$ & -48.243 & -0.359 & $0.015^{*}$ & 20.924 & 0.180 & 0.236 \\
\hline $\mathrm{F}$ & -29.365 & -0.392 & $0.008^{*}$ & 28.175 & 0.409 & $0.005^{*}$ \\
\hline S & 7.952 & 0.115 & 0.452 & -15.368 & -0.238 & 0.115 \\
\hline
\end{tabular}

Abbreviations: A, necroinflammatory activity; $F$ fibrosis, $S$ steatosis, $r A D C$ average diffusion coefficient, $F A$ fractional anisotropy

*Significant at $P<0.05$. $B$ value, unstandardized coefficient. $r$ value, partial correlation coefficient
Table 3 Results of the quantitative analysis of rADC and FA value in according to the fibrotic stage

\begin{tabular}{lll}
\hline Fibrotic stage & rADC value $\left(\times 10^{-6} \mathrm{~mm}^{2} / \mathrm{s}\right)$ & FA value $\left(\times 10^{-6} \mathrm{~mm}^{2} / \mathrm{s}\right)$ \\
\hline F0 & $899.231 \pm 109.210$ & $497.718 \pm 61.249$ \\
F1 & $835.283 \pm 129.938$ & $519.286 \pm 123.599$ \\
F2 & $802.264 \pm 61.647$ & $531.164 \pm 52.087$ \\
F3 & $786.091 \pm 70.868$ & $542.944 \pm 66.228$ \\
F4 & $716.256 \pm 72.060$ & $668.475 \pm 84.078$ \\
\hline
\end{tabular}

Abbreviations: $F$ fibrosis, $r A D C$ average diffusion coefficient, $F A$

fractional anisotropy

distinguish early cirrhosis(F4) but it has little significance in differentiating normal, mild and moderate liver fibro$\operatorname{sis}(\mathrm{F} 0 \sim \mathrm{F} 3)$.

Chronic hepatitis is characterized by a series of histological features including hepatic inflammation, liver fibrosis and fatty infiltration. As a consequence of chronic injury, the inflammatory system is first activated, which includes the activation of resident innate inflammatory cells and the recruitment of additional inflammatory cells. Then, hepatic stellate cells are activated and transformed into fibroblasts, which produce a great quantity of collagen in the extracellular matrix (ECM). The excessive deposition of ECM finally results in abnormal changes in hepatic structure and hemodynamics inside and outside of the liver [3]. When pseudolobules and nodules form, cirrhosis of the liver develops. The aggravation of hepatic inflammation is the basis of fibrosis development and the proliferation of intrahepatic fibrous septa can lead to the aggravation of hepatic inflammatory necrosis. Meanwhile, liver steatosis is also a risk factor for the progression of fibrosis. However, these abnormalities always display an otherwise normal morphology and signal in conventional MRI. With the emergence of functional MRI, the focus has changed from morphology to function [16]. For example, a gadoxetic acid enhanced MRI was used to assess liver function in cirrhosis [17]. DTI is one of the functional MRIs that can effectively detect the free diffusion rate of water molecules with different structures in vivo and can more accurately reflect changes in the direction of water molecule dispersion, which provides both functional and microstructural information for the liver through water diffusivity and diffusion anisotropy quantitation and may contribute to the evaluation of liver fibrosis [13].

The reduction in the rADC value with fibrosis observed in our study was in accordance with the findings of most prior research [18-20]. However, both our study and previous studies [11, 19] revealed that the rADC value lacked the ability to differentiating the fibrotic grades. The relationship between the FA value and liver fibrosis and the evaluation of the FA value for fibrosis staging differ. Cheung et al. [13] found that the FA value 


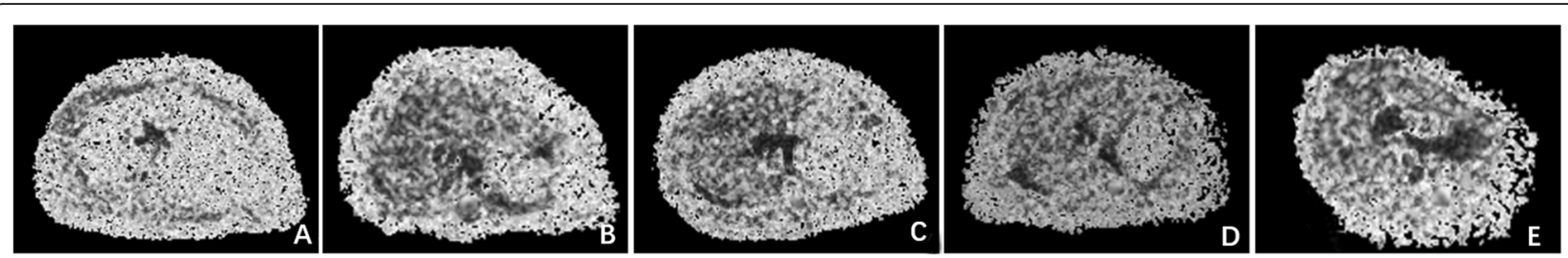

Fig. 2 FA images of the different liver fibrosis stages in rats. A E represent F0, F1, F2, F3 and F4, respectively

of rats 2 weeks after $\mathrm{CCl}_{4}$ insult was significantly lower than that before and 4 weeks after the insult, while the FA value at 4 weeks after the $\mathrm{CCl}_{4}$ insult was not significantly different from that before insult, which suggests that FA value can reveal the progression of liver fibrosis, especially early cirrhosis. Other animal research using C57BL/6 mice [12] reported that FA was negatively correlated with hepatic fibrosis and the $\operatorname{model} \operatorname{group}(n=20)$ had a lower FA than the control $\operatorname{group}(n=16)$. However, the sample size in this study was relatively small (F1 $=4$, $\mathrm{F} 2=11, \mathrm{~F} 3=5$ ), and missing value occurred for F4. In contrast, Tosun $\mathrm{M}$ et al. [11] found that the FA values showed a trend toward higher values with an increasing fibrotic stage, but there were no statistically significant differences between the FA values at the different fibrotic stages. Our study also found a positive correlation between the FA value and the fibrosis degree. Meanwhile, our study showed that the FA value of F4(early cirrhosis) was significantly different from that of F0 F3. Liver cirrhosis is the end stage of liver fibrosis which has a small chance of reversal and a high risk of developing into complications and hepatocellular carcinoma. However, radiologists cannot diagnose early cirrhosis by relying on conventional medical imaging because the morphological changes are not obvious. Our study found that the FA value of DTI can distinguish early cirrhosis, which may help physicians take early measures. One explanation for our study results is that with an increasing degree of liver fibrosis, the free movement of water molecules in the liver is affected by the presence of the fibers, and the movement direction tends to be consistent or opposite, which increases the FA value. The FA values of F0-F3 were not significantly different because in the early stages of liver fibrosis, the distribution of collagen fibers is not regular and directional, which results in the restricted diffusion of water molecules in all directions and leads to a less obvious direction of the main axis of water molecule movements. As a result, the FA value did not markedly change. With the progress of fibrosis, the fibrous bundles increased, joined into strips, flaked, and rearranged, which made the main axis of water molecule diffusion more obvious, causing the FA value to increase significantly in F4(early cirrhosis).

There are limited data regarding the relationship between liver necroinflammatory grade and DTI measurements. In general, studies $[10,11]$ found that liver ADC values were inversely correlated with inflammation. However, he rADC cannot discriminate between the different inflammation grades. Both our study and Tosun M's [11] study demonstrated that the FA value was not related to inflammation grade. The explanation for the rADC decrease with increasing inflammation grades may be the large number of inflammatory cells and factors that helped restrict the free movement ability of water molecules. However, it did not influence the movement direction of the water molecules, therefore, the FA value had no means with inflammation.

Because clinical therapy depends on the fibrotic stage and inflammatory grade, prior studies mainly used DTI for liver fibrosis and inflammation detection [10, 11]. However, some scholars emphasized that the ADC [21-24] and FA value [12] in the liver need to be carefully interpreted in the presence of hepatic steatosis. Besheer $\mathrm{T}$ [21] demonstrated that hepatic steatosis should always be considered when assessing hepatic fibrosis, and their study revealed that detected hepatic steatosis would underestimate the ADC value in patients with chronic hepatitis C. Accordingly, our study considered steatosis. However, our study results did not show a relationship between steatosis and either the $\mathrm{rADC}$ or FA value, which did not agree with the findings of some previous research [21, 24-26]. Other studies $[15,27]$ demonstrated no significant relationship between the ADC value and steatosis, which was similar to our results. The inconsistent relationship reported by researchers between MR measurements and steatosis cannot be accurately explained. It is possible that different MR machines and parameters or different group standards could affect the results.

There were limitations in our study. First, the main deficiency was that the echo-planar imaging sequence for DTI had a low signal-to-noise ratio and artifactual interference. Second, the distribution of pathological groups was uneven. The rats had individual differences in sensitivity to the induction of chronic hepatitis after injection of the same dose of drugs, which made the sample size of some groups relatively small. Future studies adopting a high signal-to-noise ratio sequence are needed. Chronic liver disease patients who have undergone hepatectomy could enroll in future studies. 


\section{Conclusion}

Our experiment showed that the $\mathrm{ADC}$ value of the DWI sequence was inversely related to hepatic fibrosis and inflammation and the FA value had a positive correlation with the degree of fibrosis. The FA value had high diagnostic accuracy in differentiating early cirrhosis and thus could be a potential marker for diagnosing liver cirrhosis. However, the rADC value lacked the ability to differentiate fibrotic grades. Overall, neither the FA nor rADC value can determine the stages of necroinflammatory activity and steatosis.

\section{Abbreviations \\ DTI: Diffusion tensor imaging; F: Liver fibrosis; A: Necroinflammatory activity; S: Steatosis; MRI: Magnetic resonance imaging; DWI: Diffusion weighted imaging; rADC: Average diffusion coefficient; FA: Fractional anisotropy; ROIs: Region of interests; ICC: Intraclass correlation coefficient; MANOVA: Multi factor analysis of variance; ANOVA: One-way analysis of variance; LSD: Least significant difference; ROC: Receiver operating characteristic curve}

\section{Acknowledgements}

Not applicable.

\section{Authors' contributions}

Contribution to conception and design: HMP, LX, WXF and SJ; Contribution to data acquisition and interpretation: HMP, LX and WXF; Contribution to performance of all statistical analyses: HMP and LX; Contribution to drafting of manuscript: HMP and LX; Contribution to critical revision of manuscript: SJ. HMP and LX had materially participated in the research and made the equal contribution, so they all ranked as the co-first authors. All authors read and approved the final manuscript.

\section{Funding}

This work was financially supported by Luzhou-Southwest Medical University Tripartite Project [grant number 2015SX-0037] for purchasing rats and producing pathological sections.

\section{Availability of data and materials}

The datasets used and analyzed during the current study are available from the corresponding author on reasonable request at shujiannc@163.com.

\section{Ethics approval and consent to participate}

All procedures performed in studies involving animals were in accordance with Guiding Principles for the Care and Use of Animals and was approved by the Animal Ethics Committee of the Southwest Medical University (Luzhou, Sichuan, China) and the laboratory animal production licenses were SCXK (Chuan) 2013-17, SCXK (Chuan) 2013-181 and SCXK (Chuan) 2013-065.

\section{Consent for publication}

Not applicable.

\section{Competing interests}

All the authors declare no competing interests.

Received: 2 April 2020 Accepted: 4 June 2020

Published online: 02 July 2020

\section{References}

1. Eom YW, Shim KY, Baik SK. Mesenchymal stem cell therapy for liver fibrosis. Korean J Intern Med. 2015;30(5):580-9.

2. Cuadrado A, Orive A, Garcia-Suarez C, Domínguez A, Fernández-Escalante JC, Crespo J, Pons-Romero F. Non-alcoholic steatohepatitis (NASH) and hepatocellular carcinoma. Obes Surg. 2005;15:442-6.

3. Elpek GO. Cellular and molecular mechanisms in the pathogenesis of liver fibrosis: an update. World J Gastroenterol. 2014;20(23):7260-76.

4. Atta HM. Reversibility and heritability of liver fibrosis: implications for research and therapy. World J Gastroenterol. 2015;21(17):5138-48.
5. Schiavon Lde L, Narciso-Schiavon JL, de Carvalho-Filho RJ. Non-invasive diagnosis of liver fibrosis in chronic hepatitis C. World J Gastroenterol. 2014; 20(11):2854-66

6. Huang D, Lin T, Wang S, Cheng L, Xie L, Lu Y, Chen M, Zhu L, Shi J. The liver fibrosis index is superior to the APRI and FIB-4 for predicting liver fibrosis in chronic hepatitis B patients in China. BMC Infect Dis. 2019;19(1):878.

7. Liang Y, Li D. Magnetic resonance elastography in staging liver fibrosis in non-alcoholic fatty liver disease: a pooled analysis of the diagnostic accuracy. BMC Gastroenterol. 2020;20(1):89.

8. Le Bihan D, Mangin JF, Poupon C, Clark CA, Pappata S, Molko N, Pappata S, Molko N. Diffusion tensor imaging concepts and applications. J Magn Reson Imaging. 2001;13(4):534-46.

9. Li Y, Zhang W. Quantitative evaluation of diffusion tensor imaging for clinical management of glioma. Neurosurg Rev. 2018. https://doi.org/10. 1007/s10143-018-1050-1.

10. Taouli B, Chouli M, Martin AJ, Qayyum A, Coakley FV, Vilgrain V. Chronic hepatitis: role of diffusion-weighted imaging and diffusion tensor imaging for the diagnosis of liver fibrosis and inflammation. J Magn Reson Imaging. 2008;28(1):89-95.

11. Tosun M, Inan N, Sarisoy HT, Akansel G, Gumustas S, Gurbuz Y, Demirci A. Diagnostic performance of conventional diffusion weighted imaging and diffusion tensor imaging for the liver fibrosis and inflammation. Eur J Radiol. 2013;82(2):203-7.

12. Lee $\mathrm{Y}, \mathrm{Kim} \mathrm{H}$. Assessment of diffusion tensor MR imaging (DTI) in liver fibrosis with minimal confounding effect of hepatic steatosis. Magn Reson Med. 2015;73(4):1602-8.

13. Cheung JS, Fan SJ, Gao DS, Chow AM, Man K, Wu EX. Diffusion tensor imaging of liver fibrosis in an experimental model. J Magn Reson Imaging. 2010;32(5):1141-8.

14. Chow AM, Gao DS, Fan SJ, Qiao Z, Lee FY, Yang J, Man K, Wu EX. Liver fibrosis: an intravoxel incoherent motion (IVIM) study. J Magn Reson Imaging. 2012;36(1):159-67.

15. Bedossa $P$, Dargere $D$, Paradis V. Sampling variability of liver fibrosis in chronic hepatitis C. Hepatology. 2003;38(6):1449-57.

16. Verde F, Romeo V, Maurea S. Advanced liver imaging using MR to predict outcomes in chronic liver disease: a shift from morphology to function liver assessment. Quant Imaging Med Surg. 2020;10(3):805-7.

17. Bastati $N$, Beer $L$, Mandorfer M, Poetter-Lang $S$, Tamandl D, Bican $Y$, Elmer MC, Einspieler H, Semmler G, Simbrunner B, Weber M, Hodge JC, Vernuccio F, Sirlin C, Reiberger T, Ba-Ssalamah A. Does the functional liver imaging score derived from gadoxetic acid-enhanced MRI predict outcomes in chronic liver disease? Radiology. 2020;294:98-107.

18. Jiang H, Chen J, Gao R, Huang Z, Wu M, Song B. Liver fibrosis staging with diffusion-weighted imaging: a systematic review and meta-analysis. Abdom Radiol (NY). 2017:42(2):490-501.

19. Li H, Chen TW, Chen XL, Zhang XM, Li ZL, Zeng NL, Zhou L, Wang LY, Tang $\mathrm{HJ}$, Li CP, Li L, Xie XY. Magnetic resonance-based total liver volume and magnetic resonance-diffusion weighted imaging for staging liver fibrosis in mini-pigs. World J Gastroenterol. 2012;18(48):7225-33.

20. Zou LQ, Chen J, Pan L, Jiang JZ, Xing W. Comparison of magnetic resonance elastography and diffusion-weighted imaging for staging hepatic fibrosis. Chin Med J. 2015;128(5):620-5.

21. Besheer T, Razek A, El Bendary M, Abd El Maksoud M, Elalfy H, Zalata K, Elkashef W, Zaghloul H, El Gilany AH. Does steatosis affect the performance of diffusion-weighted MRI values for fibrosis evaluation in patients with chronic hepatitis C genotype 4? Turk J Gastroenterol. 2017;28(4):283-8.

22. Bulow R, Mensel B, Meffert P, Hernando D, Evert M, Kuhn JP. Diffusionweighted magnetic resonance imaging for staging liver fibrosis is less reliable in the presence of fat and iron. Eur Radiol. 2013;23(5):1281-7.

23. Hansmann J, Hernando D, Reeder SB. Fat confounds the observed apparent diffusion coefficient in patients with hepatic steatosis. Magn Reson Med. 2013;69(2):545-52.

24. Poyraz AK, Onur MR, Kocakoc E, Ogur E. Diffusion-weighted MRI of fatty liver. J Magn Reson Imaging. 2012;35(5):1108-11.

25. Anderson SW, Soto JA, Milch HN, Ozonoff A, O'Brien M, Hamilton JA, Jara $\mathrm{HJ}$. Effect of disease progression on liver apparent diffusion coefficient values in a murine model of NASH at 11.7 tesla MRI. J Magn Reson Imaging. 2011;33(4):882-8.

26. Manning P, Murphy P, Wang K, Hooker J, Wolfson T, Middleton MS, Newton KP, Behling C, Awai HI, Durelle J, Paiz MN, Angeles JE, De La Pena D, 
McCutchan JA, Schwimmer JB, Sirlin CB. Liver histology and diffusionweighted MRI in children with nonalcoholic fatty liver disease: a MAGNET study. J Magn Reson Imaging. 2017;46(4):1149-58.

27. Murphy P, Hooker J, Ang B, Wolfson T, Gamst A, Bydder M, Middleton M, Peterson M, Behling C, Loomba R, Sirlin C. Associations between histologic features of nonalcoholic fatty liver disease (NAFLD) and quantitative diffusion-weighted MRI measurements in adults. J Magn Reson Imaging 2015;41(6):1629-38.

\section{Publisher's Note}

Springer Nature remains neutral with regard to jurisdictional claims in published maps and institutional affiliations.

Ready to submit your research? Choose BMC and benefit from:

- fast, convenient online submission

- thorough peer review by experienced researchers in your field

- rapid publication on acceptance

- support for research data, including large and complex data types

- gold Open Access which fosters wider collaboration and increased citations

- maximum visibility for your research: over $100 \mathrm{M}$ website views per year

At BMC, research is always in progress.

Learn more biomedcentral.com/submissions 